\title{
Parameter Optimization of Droop Controllers for Microgrids in Islanded Mode by the SQP Method with Gradient Sampling
}

\author{
Peijie Li ${ }^{1},{ }^{1}$ Ziyi Yang, ${ }^{1}$ Shuchen Huang, ${ }^{2}$ and Jun Zhang $^{3}$ \\ ${ }^{1}$ School of Electrical Engineering, Guangxi University, Nanning 530004, China \\ ${ }^{2}$ Nanning Power Supply Bureau, Guangxi Power Grid Corporation, Nanning 530031, China \\ ${ }^{3}$ Guangxi Power Grid Dispatch and Control Center, Guangxi Power Grid Corporation, Nanning 530023, China
}

Correspondence should be addressed to Peijie Li; lipeijie@gxu.edu.cn

Received 8 April 2021; Accepted 14 May 2021; Published 25 May 2021

Academic Editor: Chun Wei

Copyright (C) 2021 Peijie Li et al. This is an open access article distributed under the Creative Commons Attribution License, which permits unrestricted use, distribution, and reproduction in any medium, provided the original work is properly cited.

\begin{abstract}
For enhancing the stability of the microgrid operation, this paper proposes an optimization model considering the small-signal stability constraint. Due to the nonsmooth property of the spectral abscissa function, the droop controller parameters' optimization is a nonsmooth optimization problem. The Sequential Quadratic Programming with Gradient Sampling (SQP-GS) is implemented to optimize the droop controller parameters for solving the nonsmooth problem. The SQP-GS method can guarantee the solution of the optimization problem globally and efficiently converges to stationary points with probability of one. In the current iteration, the gradient of the nonsmooth function can be evaluated on a set of randomly generated nearby points by computing closed-form sensitivities. A test on the microgrid system shows that the optimality and the efficiency of the SQP-GS are better than those of the heuristic algorithms.
\end{abstract}

\section{Introduction}

Microgrid is an alternative solution to integrate distributed energy resources (DERs). It concludes a cluster of loads, distributed generation (DG), and energy storage systems, just like a small-scale power supply network $[1,2]$. Due to the negligible physical inertia of power-electronic converters, the microgrid operation is susceptible to random disturbances, such as variability of DG and random load fluctuations [3], like charging for electric vehicles [4], especially in an islanded mode. The small-signal stability of an islanded microgrid is critical for its reliable operation. In an islanded microgrid, droop control, following a predefined droop coefficient, is widely used to maintain the proportional load sharing between DERs [5]. The small-signal stability of the microgrid in islanded mode is strongly affected by the droop controller parameters. Therefore, it is essential to optimize the controller parameters to enhance the small-signal stability of an islanded microgrid.

Particle swarm optimization (PSO) method is often used to optimize the controller parameters of the microgrid [6-9].
In [6], the maximum of the real part of eigenvalues, also called the spectral abscissa, was minimized to enhance the stability in grid-connected mode. On the other hand, a nonlinear time-domain simulation-based objective function was employed to minimize the error in measure power in islanded mode. The study in [7] proposed a method for optimizing the controller parameters of dynamic droop control for a microgrid in islanded mode and used a spectral abscissa objective function to consider small-signal stability. The study in [8] tried to optimize proportional-integral (PI) current controller parameters considering three design criteria and multiple operating conditions which include grid-connected mode and different islanded modes by a weighted sum of multiple objective functions. The work in [9] proposed an optimization model with a nonlinear timedomain simulation-based objective function for a microgrid in islanded mode. In addition to PSO methods, the study in [10] used the Genetic Algorithms (GA) method to optimize the controller parameters by minimizing the power difference-integrating during the changing process between gridconnected mode and islanded mode. 
All the studies above adopt the heuristic algorithms to optimize the control parameters of the microgrid due to the nonsmooth property of the optimization model. Although those algorithms can obtain fast and feasible solutions, they are hard to deliver an optimal solution to the vast majority of cases $[11,12]$. The solutions derived by the heuristic algorithms are not deterministic and reproducible. These disadvantages will make the debugging and correctness checking difficult, impeding the industrial application. Concerning the optimization model, the study in [6] did not consider the small-signal stability in islanded mode. As for considering stability, the small-signal stability analysis was performed in [8-10] to obtain the optimum ranges of the controller parameters, while the study in [7] employed spectral abscissa as an objective function to enhance small-signal stability better. The study in [8] considered multiple operating conditions in the optimization model, which may cause the certain index of the design criteria to be poor due to the considering way.

To tackle the weakness mentioned above, this paper proposes an optimization model considering the spectral abscissa in multiple islanded mode operating conditions. Besides, an SQP method combined with GS (SQP-GS) [13] is presented to solve the proposed optimization model. Therefore, droop controllers with optimized parameters can enhance the small-signal stability of the microgrid in islanded mode. As for the SQP-GS method, it had been adopted to optimize the parameters of PSSs and PODs [14].

The major contributions can be summarized as follows:

(1) Based on SQP-GS, a mathematical programming method is presented to solve the proposed nonsmooth optimization model and obtain an optimal and deterministic solution without any convergence problem.

(2) The small-signal stability in different operating conditions is enhanced using a nonsmooth objective function in the optimization model. It can ensure that optimized parameters are robust under multiple operating conditions.

The remainder of this paper is organized as follows: Section 2 presents the small-signal stability model of the microgrid. Section 3 proposes the optimization model for optimizing the droop controller parameters of the microgrid. Section 4 introduces the SQP-GS method to solve the optimization model. In Section 5, the test on the microgrid system under multiple operating conditions is presented. Conclusions are drawn in Section 6.

\section{The Small-Signal Stability Model of the Microgrid}

The small-signal stability model of the microgrid can be divided into three parts: inverter, network, and load. These small-signal stability models are deduced in $\mathrm{d} q$ reference frame $[10,15]$.

2.1. The Model of the Inverter. The small-signal stability model of the inverter contains two parts: the droop controller and LC filter and coupling inductor. Moreover, the dynamic model of the droop controller consists of the power controller, voltage controller, and current controller.

2.1.1. The Model of the Power Controller. The real and reactive power can be obtained when the instantaneous active and reactive power, calculated from the measured output voltage and output current in $\mathrm{d} q$ frame, passes through the low-pass filters. The small-signal stability model can be given by

$$
\Delta \dot{P}=-\omega_{c} \Delta P+\omega_{c}\left(I_{o d} \Delta u_{o d}+I_{o q} \Delta u_{o q}+U_{o d} \Delta i_{o d}+U_{o q} \Delta i_{o q}\right),
$$

$\Delta \dot{Q}=-\omega_{c} \Delta Q+\omega_{c}\left(I_{o q} \Delta u_{o d}-I_{o d} \Delta u_{o q}-U_{o q} \Delta i_{o d}+U_{o d} \Delta i_{o q}\right)$,

where $\Delta(\cdot)$ is the state variable with respect to the time, $\Delta(\cdot)$ is the state variable or the algebraic variable after linearization, $P, Q$ are the real and reactive power, respectively, $u_{o d}, u_{o q}, i_{o d}, i_{o q}$ are the output voltage and current in $\mathrm{d} q$ frame, respectively, and $\omega_{c}$ is the cut-off frequency of the low-pass filters.

The frequency and voltage can be set by the droop gain; and the small-signal stability model can be given by

$$
\begin{aligned}
\Delta \omega & =-m_{p} \Delta P, \\
\Delta u_{o d}^{*} & =-n_{q} \Delta Q, \\
\Delta u_{o q}^{*} & =0,
\end{aligned}
$$

where $\omega$ is the frequency, $m_{p}, n_{q}$ are the droop gains related to frequency and voltage amplitude, respectively, and $u_{o d}^{*}, u_{o q}^{*}$ are the output voltage reference in $\mathrm{d} q$ frame, respectively.

The first $\mathrm{d} q$ frame of the inverter is set as the common frame; and all the variables from other inverter frames can be converted to the common frame. The small-signal stability model is given by

$$
\Delta \dot{\delta}=\Delta \omega-\Delta \omega_{\text {com }}=-m_{p} \Delta P-\Delta \omega_{\text {com }}
$$

where $\omega_{\text {com }}$ is the frequency of the common frame taken by the first inverter and $\delta$ is the angle between an inverter frame and the common frame.

2.1.2. The Model of the Voltage Controller. The voltage controller contains the PI regulator and the feed-forward terms; and the small-signal stability model of the voltage controller can be given by

$$
\begin{aligned}
& \Delta \dot{\phi}_{d}=\Delta u_{o d}^{*}-\Delta u_{o d} \\
& \Delta \dot{\phi}_{q}=\Delta u_{o q}^{*}-\Delta u_{o q} \\
& \Delta i_{l d}^{*}=K_{i u} \Delta \phi_{d}+K_{p u}\left(\Delta u_{o d}^{*}-\Delta u_{o d}\right)-\omega_{n} C_{f} \Delta u_{o q}+F \Delta i_{o d}
\end{aligned}
$$




$$
\Delta i_{l q}^{*}=K_{i u} \Delta \phi_{q}+K_{p u}\left(\Delta u_{o q}^{*}-\Delta u_{o q}\right)+\omega_{n} C_{f} \Delta u_{o d}+F \Delta i_{o q}
$$

where $\phi_{d}, \phi_{q}$ are $\int\left(u_{o d}^{*}-u_{o d}\right) \mathrm{d} t$ and $\int\left(u_{o q}^{*}-u_{o q}\right) \mathrm{d} t$, respectively, $i_{l d}^{*}, i_{l q}^{*}$ are the output current reference in $\mathrm{d} q$ frame, respectively, $K_{i u}, K_{p u}$ are the integral and proportional gains of the PI regulator in voltage controller, respectively, $w_{n}$ is the nominal frequency, $F$ is the feedforward gain, and $C_{f}$ is the per-phase capacitance.

2.1.3. The Model of the Current Controller. The current controller includes the PI regulator; and the small-signal stability model of the current controller can be given by

$$
\begin{aligned}
\Delta \dot{\gamma}_{d} & =\Delta i_{l d}^{*}-\Delta i_{l d}, \\
\Delta \dot{\gamma}_{q} & =\Delta i_{l q}^{*}-\Delta i_{l q}, \\
\Delta u_{i d}^{*} & =K_{i c} \Delta \gamma_{d}+K_{p c}\left(\Delta i_{l d}^{*}-\Delta i_{l d}\right)-\omega_{n} L_{f} \Delta i_{l q}, \\
\Delta u_{i q}^{*} & =K_{i c} \Delta \gamma_{q}+K_{p c}\left(\Delta i_{l q}^{*}-\Delta i_{l q}\right)+\omega_{n} L_{f} \Delta i_{l d},
\end{aligned}
$$

where $\gamma_{d}, \gamma_{q}$ are $\int\left(i_{l d}^{*}-i_{l d}\right) \mathrm{d} t$ and $\int\left(i_{l q}^{*}-i_{l q}\right) \mathrm{d} t$, respectively, $u_{i d}^{*}, u_{i q}^{*}$ are the output inverter voltage reference in $\mathrm{d} q$ frame, respectively, $i_{l d}, i_{l q}$ are the sampled filter current in $\mathrm{d} q$ frame, respectively, $K_{i c}, K_{p c}$ are the integral and proportional gains in PI regulator of the current controller, respectively, and $L_{f}$ is the per-phase inductance.

2.1.4. The Model of the LC Filter and Coupling Inductance. The LC filter and coupling inductance can remove the harmonic wave near the switch frequency; and the smallsignal stability model of the LC filter and coupling inductance are given by

$$
\begin{aligned}
& \Delta \dot{i}_{l d}=-\frac{R_{f}}{L_{f}} \Delta i_{l d}+\omega_{o} \Delta i_{i q}-\frac{1}{L_{f}} \Delta u_{o d}+\frac{1}{L_{f}} \Delta u_{i d}+I_{l q} \Delta \omega, \\
& \Delta \dot{i}_{l q}=-\frac{R_{f}}{L_{f}} \Delta i_{l q}-\omega_{o} \Delta i_{i d}-\frac{1}{L_{f}} \Delta u_{o q}+\frac{1}{L_{f}} \Delta u_{i q}-I_{l d} \Delta \omega, \\
& \Delta \dot{u}_{o d}=\frac{1}{C_{f}} \Delta i_{l d}+\omega_{o} \Delta u_{o q}-\frac{1}{C_{f}} \Delta i_{o d}+U_{o q} \Delta \omega, \\
& \Delta \dot{u}_{o q}=\frac{1}{C_{f}} \Delta i_{l q}-\omega_{o} \Delta u_{o d}-\frac{1}{C_{f}} \Delta i_{o q}-U_{o d} \Delta \omega, \\
& \Delta \dot{i}_{o d}=\frac{1}{L_{c}} \Delta u_{o d}-\frac{R_{c}}{L_{c}} \Delta i_{o d}+\omega_{o} \Delta i_{o q}-\frac{1}{L_{c}} \Delta u_{b d}+I_{o q} \Delta \omega, \\
& \Delta \dot{i}_{o q}=\frac{1}{L_{c}} \Delta u_{o q}-\frac{R_{c}}{L_{c}} \Delta i_{o q}-\omega_{o} \Delta i_{o d}-\frac{1}{L_{c}} \Delta u_{b q}-I_{o d} \Delta \omega,
\end{aligned}
$$

where $u_{b d}, u_{b q}$ are the bus voltage in $\mathrm{d} q$ frame, respectively, $u_{i d}, u_{i q}$ are the inverter voltage in $\mathrm{d} q$ frame, respectively, $\omega_{0}, I_{l d}, I_{l q}, U_{o d}, U_{o q}, I_{o d}, I_{o q}$ are the steady state value at the initial operating point, and $R_{f}, R_{c}, L_{f}, L_{c}, C_{f}$ are the resistance, inductance, and capacitance in the microgrid, respectively.

2.1.5. The Model of the Individual Inverter. Through the transformation matrix, other inverter variables can transfer to the common frame. The linearization models of the transformation matrix are obtained:

$$
\begin{aligned}
{\left[\Delta i_{o D Q i}\right]=} & {\left[\begin{array}{cc}
\cos \delta_{o} & -\sin \delta_{o} \\
\sin \delta_{o} & \cos \delta_{o}
\end{array}\right]\left[\Delta i_{o d q i}\right] } \\
& +\left[\begin{array}{c}
-I_{o d} \sin \delta_{o}-I_{o q} \cos \delta_{o} \\
I_{o d} \cos \delta_{o}-I_{o q} \sin \delta_{o}
\end{array}\right]\left[\Delta \delta_{i}\right], \\
{\left[\Delta u_{b d q i}\right]=} & {\left[\begin{array}{cc}
\cos \delta_{o} & \sin \delta_{o} \\
-\sin \delta_{o} & \cos \delta_{o}
\end{array}\right]\left[\Delta u_{b D Q i}\right] } \\
& +\left[\begin{array}{c}
-U_{b D} \sin \delta_{o}+U_{b Q} \cos \delta_{o} \\
-U_{b D} \cos \delta_{o}-U_{b Q} \sin \delta_{o}
\end{array}\right]\left[\Delta \delta_{i}\right],
\end{aligned}
$$

where $\Delta i_{o D Q i}, \Delta u_{b D Q i}$ are the output current and bus voltage of the $i$ th inverter converted to the common frame, respectively, and $\delta_{0}$ is the angle between the inverter frame and the common frame.

Combined by the linearization models (1)-(22), the linearization state equation and the linearization output equation of the $i$ th inverter in the common frame are given by

$$
\left[\Delta \dot{x}_{i n v i}\right]=A_{I N V_{i}}\left[\Delta x_{i n v i}\right]+B_{I N V i}\left[\Delta u_{b D Q i}\right]+B_{i \omega c o m}\left[\Delta \omega_{c o m}\right],
$$

$$
\left[\begin{array}{c}
\Delta \omega_{i} \\
\Delta i_{o D Q i}
\end{array}\right]=\left[\begin{array}{c}
C_{I N V \omega i} \\
C_{I N V c i}
\end{array}\right]\left[\Delta x_{i n v i}\right]
$$

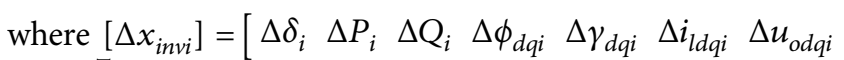
$\left.\Delta i_{\text {odqi }}\right]^{T}$.

2.2. The Model of the Network and Load. The linearization models of the network and load are obtained:

$$
\begin{aligned}
{\left[\Delta \dot{i}_{\text {line } D Q}\right]=} & A_{\mathrm{NET}}\left[\Delta i_{\text {line } D \mathrm{Q}}\right]+B_{1 \mathrm{NET}}\left[\Delta u_{b D \mathrm{Q}}\right]+B_{2 \mathrm{NET}} \Delta \omega, \\
{\left[\Delta \dot{i}_{\text {loadDQ }}\right]=} & A_{\mathrm{LOAD}}\left[\Delta i_{\text {loadDQ }}\right]+B_{1 \mathrm{LOAD}}\left[\Delta u_{b \mathrm{DQ}}\right] \\
& +B_{2 \mathrm{LOAD}} \Delta \omega,
\end{aligned}
$$

where $\quad \Delta i_{\text {line } D Q}=\left[\Delta i_{\text {line } D Q 1}, \Delta i_{\text {line } D Q 2}, \ldots, \Delta i_{\text {line } D Q n}\right]^{T}$, $\Delta i_{\text {load } D Q}=\left[\Delta i_{\text {load } D Q 1}, \Delta i_{\text {load } D Q 2}, \ldots, \Delta i_{\text {load } D Q p}\right]^{T}$, and $n, p$ are the numbers of the lines and loads, respectively.

2.3. The Model of the Microgrid. To define the node voltage clearly, the virtual resistor $R_{N}$ is assumed between each node and ground. The small-signal stability model is given by 


$$
\left[\Delta u_{b \mathrm{DQ}}\right]=R_{N}\left(M_{\mathrm{INV}}\left[\Delta i_{o D Q}\right]+M_{\mathrm{LOAD}}\left[\Delta i_{\text {loadDQ }}\right]+M_{\mathrm{NET}}\left[\Delta i_{\text {lineDQ }}\right]\right),
$$

where $\Delta \mathfrak{u}_{b D Q}=\left[\Delta u_{b D Q 1}, \Delta u_{b D Q 2}, \ldots, \Delta u_{b D Q m}\right]^{T}, m$ is the number of nodes in the microgrid, $M_{\mathrm{INV}}$ maps the inverter connection points onto network nodes, $M_{\mathrm{LOAD}}$ maps the load connection point onto the network nodes, and $M_{\mathrm{NET}}$ maps the connection lines onto nodes.

Combined with (23)-(27), the small-signal stability model of the microgrid is given by

$$
\left[\begin{array}{c}
\Delta \dot{x}_{\mathrm{INV}} \\
\Delta \dot{i}_{\text {line } D Q} \\
\Delta \dot{i}_{\text {load } D Q}
\end{array}\right]=A_{m g}\left[\begin{array}{c}
\Delta x_{\mathrm{INV}} \\
\Delta \dot{i}_{\text {line } D Q} \\
\Delta \dot{i}_{\text {load } D Q}
\end{array}\right],
$$

where $\Delta x_{\mathrm{INV}}=\left[x_{\mathrm{INV} 1}, x_{\mathrm{INV} 2}, \ldots, x_{\mathrm{INV} s}\right]^{T}, s$ is the number of inverters, and $A_{m g}$ is the state matrix of the microgrid model.

\section{The Proposed Optimization Model}

The dynamic system is stable in small-signal stability sense when all the real parts of the eigenvalues of $A_{m g}$ are negative. The largest real part of all eigenvalues is called the spectral abscissa $\eta$, which stands for the power system's stability margin. The value of the spectral abscissa is smaller, and the rate of decay is faster when the system suffers a small disturbance. Therefore, the dynamic system is more stable in the small-signal stability sense. Based on the above smallsignal stability model of microgrid, the eigenvalues of $A_{m g}$ can be obtained. Therefore, the spectral abscissa $\eta$ can be given by

$$
\eta\left(\mathbf{A}_{\mathbf{m g}}\right)=\max \left\{\operatorname{Re}(\lambda): \lambda \in \lambda\left(\mathbf{A}_{\mathbf{m g}}\right)\right\}=\operatorname{Re}\left(\lambda_{\eta}\right),
$$

where $\lambda\left(\mathbf{A}_{\mathbf{m g}}\right)$ represents all eigenvalues of $\mathbf{A}_{\mathbf{m g}} \cdot \operatorname{Re}(\lambda)$ is the real part of all eigenvalues, and $\lambda_{\eta}$ is the eigenvalue with largest real part.

Since the load model is contained in the small-signal stability model of the microgrid, the variation of the load can affect the spectral abscissa of the microgrid exceedingly. Also, load condition variation, such as random load fluctuation, frequently happens in the microgrid operation. Although the microgrid can work well at one load condition, also called operating conditions, it cannot guarantee the small-signal stability at other operating conditions. Therefore, it is crucial to consider multiple operating conditions in the proposed optimization model to achieve better robustness.

The spectral abscissas of different operating condition can be represented as $\eta_{i}$, which stands for the spectral abscissa of the $i$ th operating conditions, $i=[1,2, \ldots, l]$, and $l$ is the number of the operating conditions.

In $[7,9,15]$, through the eigenvalue analysis, the dominant eigenvalues near the imaginary axis are largely sensitive to $m_{p}, n_{q}$ and the eigenvalues of medium frequency are largely sensitive to $K_{p u}, K_{i u}, K_{p c}$, and $K_{i c}$. The value of $\eta$, even the system stability, will vary with droop controller parameters.

Therefore, the critical droop controller parameters of each inverter are considered as the variables $\mathbf{x}$ to be optimized:

$$
x=\left[m_{p 1}, n_{q 1}, K_{p u 1}, K_{i u 1}, K_{p c 1}, K_{i c 1}, \ldots, m_{p 2}, n_{q 2}, K_{p u 2}, K_{i u 2}, K_{p c 2}, K_{i c 2}, \ldots, m_{p N}, n_{q N}, K_{p u N}, K_{i u N}, K_{p c N}, K_{i c N}\right]
$$

where $N$ is the total number of inverters.

According to (29) and (30), the spectral abscissa is actually an implicit function of the controller parameters:

$$
\eta_{i}=f\left(m_{p}, n_{q}, K_{p u}, K_{i u}, K_{p c}, K_{i c}, \ldots, m_{p N}, n_{q N}, K_{p u N}, K_{i u N}, K_{p c N}, K_{i c N}\right) .
$$

The spectral abscissas of the worst operating condition, also called the largest spectral abscissa, can be represented as $\eta_{\max }$ :

$$
\eta_{\max }=\max \left\{\eta_{1}, \eta_{2}, \ldots, \eta_{l}\right\} .
$$

According to (32), the objective function can be given to achieve maximum stability of the microgrid in the worst operating condition. This objective function also ensures the maximum stability of microgrid in other operating conditions:

$$
\min \eta_{\max }
$$

The constraints of controller parameters of the $k$ th inverter can be listed as the constraint of optimization model:

$$
\begin{aligned}
\underline{m}_{p k} & \leq m_{p k} \leq \bar{m}_{p k}, \\
\underline{n}_{q k} & \leq n_{q k} \leq \bar{n}_{q k}, \\
\underline{K}_{p u k} & \leq K_{p u k} \leq \bar{K}_{p u k}, \\
\underline{K}_{i u k} & \leq K_{i u k} \leq \bar{K}_{i u k}, \\
\underline{K}_{p c k} & \leq K_{p c k} \leq \bar{K}_{p c k}, \\
\underline{K}_{i c k} & \leq K_{i c k} \leq \bar{K}_{i c k} .
\end{aligned}
$$

However, $\eta_{\max }$ is not locally Lipschitz. Fortunately, $\eta_{i}$ has been proved to be locally Lipschitz and continuously differentiable on open dense subsets of $\mathbb{R}^{n}[16]$, which means that it is continually differentiable almost everywhere. Also, its gradient can be obtained by calculating spectral abscissa sensitivities. Besides, the small-signal stability constraint with the spectral abscissa of multiple operating conditions can be added to the optimization model to ensure that the microgrid works well in the multiple operating conditions; and, due to the small-signal stability constraint in the optimization model, minimizing $\bar{\eta}$ is equivalent to minimize $\eta_{\max }$. 
Through the above discussion, the complete optimization model can be given by

$$
\min \bar{\eta}
$$

where $\bar{\eta}$ is the upper limit of spectral abscissa; and the constraints of the optimization model can be listed as follows:

$$
\begin{gathered}
\eta_{i} \leq \bar{\eta}, \\
\underline{m}_{p k} \leq m_{p k} \leq \bar{m}_{p k}, \\
\underline{n}_{q k} \leq n_{q k} \leq \bar{n}_{q k}, \\
\underline{K}_{p u k} \leq K_{p u k} \leq \bar{K}_{p u k}, \\
\underline{K}_{i u k} \leq K_{i u k} \leq \bar{K}_{i u k}, \\
\underline{K}_{p c k} \leq K_{p c k} \leq \bar{K}_{p c k}, \\
\underline{K}_{i c k} \leq K_{i c k} \leq \bar{K}_{i c k},
\end{gathered}
$$

where $\overline{(\cdot)}$ and $(\cdot)$ are the upper and lower limits of controller parameters and $k=[1,2, \ldots, N]$.

The spectral abscissa of multiple operating conditions moves toward the left as far as possible to ensure maximum stability through the optimization model. Thus, the microgrid with optimized controller parameters can operate stably in multiple operating conditions.

According to (31), the spectral abscissa cannot be deduced as an analytical and nonlinear or linear expression of controller parameters; and the spectral abscissa with respect to the parameters has been proved to be nonsmooth [17]. The optimization model in (35)-(42) is a nonsmooth programming problem, which cannot be tackled by the methods for linear or nonlinear programming. It is hard to solve the nonsmooth programming in mathematical programming until the breakthrough in [13].

\section{The SQP-GS Method for Solving the Optimization Problem}

4.1. The SQP-GS Method. According to the Clarke theorem, if any subgradient is zero, which belongs to the subdifferential of $x_{0}$, there is a stationary point $x_{0}$ in this nonsmooth function [18].

The nonsmooth problem is hard to be solved until a GS method was proposed in 2005 [18], which can approximate the subgradients for minimizing a nonsmooth objective function. In [18], if the nonsmooth function or constraints are locally Lipschitz continuous, a series of the independent and random gradients of sample point near the nonsmooth point $x$ can be evaluated as convex hull to express the subgradients of $x$.

The traditional SQP method can solve a QP subproblem to obtain a descending direction. It only solves the smooth problem but fails for the nonsmooth problem. In 2012, the Sequential Quadratic Programming with Gradient Sampling (SQP-GS) method was proposed in [13]. It can converge globally to stationary points with probability of one when the objective function or constraints are locally Lipschitz and continuously differentiable on open dense subsets of $\mathbb{R}^{n}$.

The SQP-GS method is developed to solve the optimization model in the following form:

$$
\begin{array}{lr} 
& \min _{\mathbf{x}} f(\mathbf{x}) \\
\text { s.t. } & \mathbf{g}(\mathbf{x}) \leq 0,
\end{array}
$$

where the objective function $f: \mathbb{R}^{n} \longrightarrow \mathbb{R}$ and the inequality constraint functions $\mathbf{g}: \mathbb{R}^{n} \longrightarrow \mathbb{R}^{m}$.

The SQP-GS method can compute a search direction $\mathbf{d}_{k}$ in the $k$ th iteration by solving a QP dual subproblem:

$$
\begin{gathered}
\min _{\mathbf{d}_{k}, \mathbf{y}_{f}, \mathbf{y}_{g}}\left[\begin{array}{r}
-f\left(\mathbf{x}_{k}\right) \\
-\mathbf{g}\left(\mathbf{x}_{k}\right)
\end{array}\right]^{\top}\left[\begin{array}{l}
\mathbf{y}_{f} \\
\mathbf{y}_{g}
\end{array}\right]+\left[\mathbf{d}_{k}^{\top}, \mathbf{y}_{f}^{\top}, \mathbf{y}_{g}^{\top}\right] \mathbf{H}_{k}\left[\begin{array}{l}
\mathbf{d}_{k} \\
\mathbf{y}_{f} \\
\mathbf{y}_{g}
\end{array}\right] \\
\text { s.t. } \quad \mathbf{H}_{k} \mathbf{d}_{k}+\nabla f\left(\mathbf{x}^{f}\right)^{T} \mathbf{y}_{f}+\nabla \mathbf{g}\left(\mathbf{x}^{g}\right)^{T} \mathbf{y}_{g}=0 \\
\forall \mathbf{x}^{f} \in \mathscr{B}_{\epsilon, k}^{f}, \forall \mathbf{x}^{g} \in \mathscr{B}_{\epsilon, k}^{g}, \\
e^{T} \mathbf{y}_{f}=\rho \\
e^{T} \mathbf{y}_{g}=1 \\
\mathbf{y}_{f} \geq 0 \\
\mathbf{y}_{g} \geq 0
\end{gathered}
$$

where $\mathbf{x}_{k}$ is the vector of variables in the $k$ th iteration, $\mathbf{H}_{k}$ is the approximate Hessian of the Lagrangian optimization model of (43), $\rho$ is a penalty parameter, and $\mathbf{y}_{f}=\left(y_{k, 0}^{f}, y_{k, 1}^{f}, \ldots, y_{k, p}^{f}\right)$ and $\mathbf{y}_{g}=\left(y_{k, 0}^{g_{j}}, y_{k, 1}^{g_{j}}, \ldots, y_{k, p}^{g_{j}}\right)$ are the approximated minimum-norm elements of the subdifferential of $f\left(\mathbf{x}_{k}\right)$ and $\mathbf{g}\left(\mathbf{x}_{k}\right)$, respectively, $\mathbf{x}^{f}$ and $\mathbf{x}^{g}$ are the vectors of the sample points of $f\left(\mathbf{x}_{k}\right)$ and $\mathbf{g}\left(\mathbf{x}_{k}\right)$, respectively, and

$$
\begin{array}{ll}
\mathscr{B}_{\epsilon, k}^{f}:=\left\{x_{k, 0}^{f}, x_{k, 1}^{f}, \ldots, x_{k, p}^{f}\right\}, & \text { where } x_{k, 0}^{f}:=x_{k}, \\
\mathscr{B}_{\epsilon, k}^{g^{j}}:=\left\{x_{k, 0}^{g^{j}}, x_{k, 1}^{g^{j}}, \ldots, x_{k, p}^{g^{j}}\right\}, & \text { where } x_{k, 0}^{g^{j}}:=x_{k},
\end{array}
$$

are sets of independent and identically distributed random sample points sampled from the closed ball $\mathbb{B}_{\epsilon}\left(\mathbf{x}_{k}\right)$ centered at $\mathbf{x}_{k}$, and

$$
\mathbb{B}_{\epsilon}\left(\mathbf{x}_{k}\right)=\left\{\mathbf{x} \mid\left\|\mathbf{x}-\mathbf{x}_{k}\right\| \leq \varepsilon\right\}
$$

For reflecting the iteration process, infeasibility value can be defined as

$$
\sigma_{k}\left(\mathbf{x}_{k}\right):=\max \left(\mathbf{g}\left(\mathbf{x}_{k}\right), 0\right) .
$$

The following model reduction $\Delta \mathbf{q}_{k}$ is also defined in terms of primal and dual infeasibility: 


$$
\Delta \mathbf{q}_{k}=\rho f\left(\mathbf{x}_{k}\right)+\sigma_{k}\left(\mathbf{x}_{k}\right)-\rho \max _{\mathbf{x}_{k} \in \mathscr{B}_{\epsilon, k}^{f}}\left\{f\left(\mathbf{x}_{k}\right)+\nabla f\left(\mathbf{x}_{k}\right)^{\top} \mathbf{d}_{k}\right\}-\sum_{j=1}^{m} \max _{\mathbf{x}_{k} \in \mathscr{B}_{\epsilon, k}^{g, k}}\left\{\max \left\{g^{j}\left(\mathbf{x}_{k}\right)+\nabla g^{j}\left(\mathbf{x}_{k}\right)^{\top} \mathbf{d}_{k}, 0\right\}\right\}-\frac{1}{2} \mathbf{d}_{k}^{\top} \mathbf{H}_{k} \mathbf{d}_{k} .
$$

When the SQP-GS method converges to the optimal solution, these optimality certificates, such as $\sigma_{k}\left(\mathbf{x}_{k}\right)$, $\Delta \mathbf{q}_{k}$, can be 0 . The sample size $p$ can be set to 0 when the SQP-GS method is solving a smooth function, in which case the SQP-GS method reduces to the general SQP method [14].

4.2. Gradient Evaluation of Spectral Abscissa Function. The gradient of the spectral abscissa $\nabla f(x)$ is crucial for implementing the SQP-GS method; and the spectral abscissa with respect to the controller parameters, also called the spectral abscissa sensitivity, reflects the variation of the variable $\mathbf{x}$ on the impact of system stability. Therefore, the gradient $\nabla f(x)$ of spectral abscissa can be represented as spectral abscissa sensitivity. The spectral abscissa sensitivity can be calculated by the numerical differentiation method, which is numerical spectral abscissa sensitivity.

The numerical spectral abscissa sensitivity can be obtained by

$$
\frac{\partial \eta}{\partial \mathbf{x}} \approx \frac{\eta\left(\mathbf{A}_{\varepsilon}\right)-\eta(\mathbf{A})}{\varepsilon}
$$

where $\eta(\mathbf{A})$ is the spectral abscissa of the state matrix at the equilibrium point, $\varepsilon$ is the small quantity of the variable variation, $\mathbf{x}$ is the variable, and $\eta\left(\mathbf{A}_{\varepsilon}\right)$ is the spectral abscissa of perturbed state matrix.

As for different variables, the small quantity range is not defined clearly. It causes the accuracy of numerical spectral abscissa sensitivity to be uncertain. When calculating the spectral abscissa sensitivity for each variable, the eigenvalue of $\mathbf{A}_{\varepsilon}$ needs to be recalculated more than once in each iteration. It decreases the efficiency of the optimization.

Hence, the closed-form eigenvalue sensitivity can be an alternative option [19]. It is a mathematical eigenvalue derived at an equilibrium point; and the eigenvalue sensitivity with respect to variable $\mathbf{x}$ can be expressed as

$$
\frac{\partial \lambda_{i}}{\partial \mathbf{x}}=\frac{\psi_{i}^{\top}\left(\left(\partial\left(\mathbf{A}_{m g}\right)\right) / \partial \mathbf{x}\right) \phi_{i}}{\psi_{i}^{\top} \phi_{i}}
$$

where $\mathbf{A}_{m g}$ is the state matrix of microgrid at the equilibrium point, $\psi_{i}^{\top}$ and $\phi_{i}$ are the left and right eigenvectors of $\lambda_{i}$ at an equilibrium point, respectively, and $\left(\partial \mathbf{A}_{m g} / \partial \mathbf{x}\right)$ can be obtained on matrix calculus.

The closed-form spectral abscissa sensitivity can be expressed by

$$
\frac{\partial \eta}{\partial x}=\operatorname{Re}\left(\frac{\partial \lambda_{\eta}}{\partial x}\right)
$$

In (56) and (57), $\psi_{\eta}^{\top}, \phi_{\eta}$, and $\left(\partial \mathbf{A}_{m g} / \partial \mathbf{x}\right)$ are required to calculate only once in each iteration. Besides, the closed- form spectral abscissa sensitivity is more accurate than numerical spectral abscissa sensitivity in each iteration. Therefore, the closed-form spectral abscissa sensitivity is used to compute the gradient of the spectral abscissa.

4.3. Solving the Droop Controller Parameters Optimization Problem by SQP-GS. Before employing the SQP-GS method to solve a QP subproblem to get a descending direction, the gradients of objective function and constraints in the optimization model with respect to variable $\mathbf{x}$ should be obtained. The constraint functions in (37)(42) are smooth; and the gradient of these constraints can be obtained easily. Therefore, the sample size $p$ reduces to zero when the SQP-GS method solves the smooth function. Objective function (35) and the small-signal stability constraint (36) are nonsmooth. The state matrix $A_{m g}$ in (28) for each point in $\mathscr{B}_{\epsilon, k}^{g^{j}}$ is established. The most critical eigenvalue $\lambda_{\eta}$ and the corresponding left and right eigenvectors $\psi_{i}^{\top}$ and $\phi_{i}^{\top}$ for each $A_{m g}$ can be obtained in each iteration. According to (56), the most critical eigenvalue sensitivity $\left(\partial \lambda_{\eta} / \partial x\right)$ with respect to the $i$ th variable for each point is calculated. Therefore, the gradient $(\partial \eta / \partial x)$ with respect to the $i$ th variable in (57) for each point can be obtained. The initial values of droop controller parameters need to be set in the range of controller parameters limit. Otherwise, they may not converge. The Algorithm 1 is given as follows [14].

\section{Test Variation}

The following test system in Figure 1 is used to demonstrate the effectiveness of the proposed SQP-GS method. The simulations are carried out on a computer with a fourcore $3.70 \mathrm{GHz}$ processor and $16 \mathrm{~GB}$ RAM. Also, the SQPGS method is implemented in MATLAB R2012a. As for the SQP-GS method, those parameters are selected as follows: $\rho=5 e-4, \mu_{\rho}=0.5, \varepsilon=0.1, \mu_{\varepsilon}=0.1, \theta=0.1$, and $\mu_{\theta}=0.8$.

5.1. Microgrid System Parameters and Four Operating Conditions. We design four operating conditions of the microgrid to verify the effectiveness of the SQP-GS method. The test system in Figure 1 contains three inverters, two connection lines, and two loads. Load 1 is in bus 1 , and the other one is in bus 3; and the microgrid system parameters are given in Table 1. Also, the initial condition of the microgrid system is given in Table 2 .

To ensure that the droop controller parameters are robust after optimization, similar to multiple operating conditions in [6], we design different operating conditions. They are shown in Table $3 . R_{\text {load }}$ of $25 \Omega$ and $20 \Omega$ per phase is equal to the resistive load of $5.6 \mathrm{~kW}$ and $7.3 \mathrm{~kW}$ [13]. The objection of this paper is to investigate the optimized 
(1) Initialization: set $k=1, k_{\max }=200$; Set sample size $p>0$, sample radius $\varepsilon>0$, constraint violation tolerance $\theta>0$, penalty parameter $\rho>0$, line search constant $\emptyset \in(0,1)$, backtracking constant $\gamma \in(0,1)$, sampling radius reduction factor $\mu_{\varepsilon} \in(0,1)$, constraint violation tolerance reduction factor $\mu_{\theta} \in(0,1)$, penalty parameter reduction factor $\mu_{\rho} \in(0,1)$, infeasibility tolerance $v_{\text {in }}>0$, stationarity tolerance parameter $\nu_{s}>0$.

(2) Termination conditions check: while $k<k_{\max }$, if $\max \left(\Delta \mathbf{q}_{k}\right)<v_{s}$ or $\varepsilon<v_{s}$, and $\sigma_{k}<v_{i n}$, output solution and stop.

(3) Gradient sampling: generate sample point $\mathscr{B}_{\varepsilon, k}^{f}$ in (50) and obtain gradient of multiple operating condition $\left(\partial \eta_{i} / \partial \mathbf{x}\right)$ from (56) and (57), Generate sample point $\mathscr{B}_{\varepsilon, k}^{g}$ from (51), and get the gradient of smooth constraint in (37)-(42).

(4) Search direction calculation: set $H_{k}>0$, solve (45)-(49) to get $\left(\mathbf{d}_{k}, \mathbf{y}_{f}, \mathbf{y}_{g}\right)$.

(5) L-BFGS update: update $H_{k}$ for next iteration according to Limited-memory Broyden-Fletcher-Goldfarb-Shanno (L-BFGS) method [20].

(6) Parameter update: if $\Delta \mathbf{q}_{k}>v_{s} \varepsilon^{2}$, go to Step 7. Otherwise, if $\sigma_{k} \leq \theta$, set $\theta \leftarrow \mu_{\theta} \theta$; if $\sigma_{k}>\theta$, set $\rho \leftarrow \mu_{\rho} \rho$. And set $\varepsilon \leftarrow \mu_{\varepsilon} \varepsilon, \beta_{k} \leftarrow 0$ and go to step 8.

(7) Line search: set $\beta_{k}$ as the largest value in the sequence $\left\{1, \gamma, \gamma^{2}, \ldots\right\}$ such that $\mathbf{x}_{k+1} \leftarrow \mathbf{x}_{k}+\beta_{k} \mathbf{d}_{k} \quad$ satisfies: $\rho f\left(\mathbf{x}_{k+1}\right)+\sigma\left(\mathbf{x}_{k+1}\right) \leq \rho f\left(\mathbf{x}_{k}\right)+\sigma\left(\mathbf{x}_{k}\right)-\Phi \beta_{k} \Delta \mathbf{q}_{k}$

(8) Iteration increment: set $k \leftarrow k+1$ and go to step 2 .

(9) end do

AlgORITHM 1: SQP-GS method.

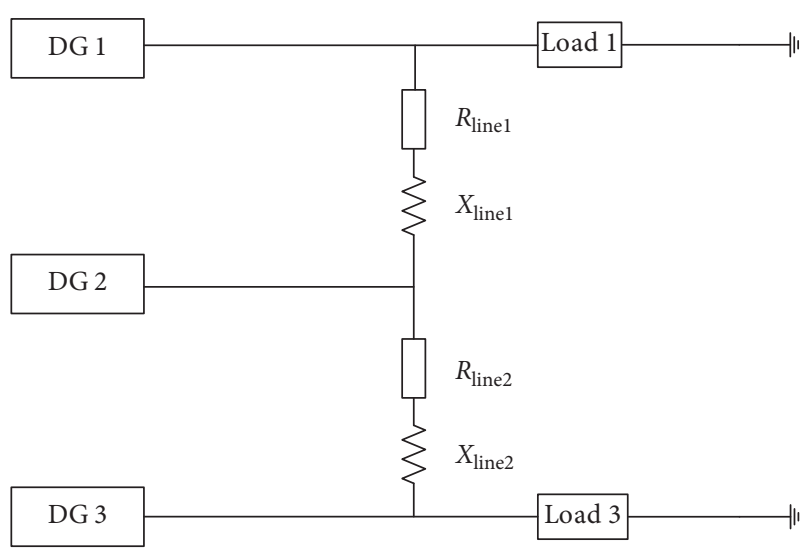

Figure 1: Microgrid structure.

TABLE 1: The microgrid system parameters.

\begin{tabular}{lc}
\hline Parameter & Value \\
\hline$L_{f}$ & $1.35 e-3 H$ \\
$C_{f}$ & $50 e-6 F$ \\
$R_{f}$ & $0.1 \Omega$ \\
$R_{\text {line1 }}$ & $0.23 \Omega$ \\
$R_{\text {line2 }}$ & $0.35 \Omega$ \\
$L_{c}$ & $0.35 e-3 H$ \\
$R_{c}$ & $0.03 \Omega$ \\
$F$ & 0.75 \\
$L_{\text {line1 }}$ & $3.2 e-4 H$ \\
$L_{\text {line2 }}$ & $1.9 e-3 H$ \\
\hline
\end{tabular}

parameters in droop controllers that can enhance the smallsignal stability of all four operating conditions.

The limit of controller parameters can be shown in Table 4. It is selected based on the trace of the eigenvalues varying with the controller parameters variation $[9,10]$. When the droop controller parameters vary in the limit of the variable, the critical eigenvalue will move within the stable region. Meanwhile, the stability of the microgrid operation can be guaranteed. According to the above rule,
TABLE 2: The initial condition of the microgrid system.

\begin{tabular}{lc}
\hline Parameter & Value \\
\hline$V_{o d}$ & {$[380.8,381.8,380.4]$} \\
$I_{o d}$ & {$[11.4,11.4,11.4]$} \\
$I_{l d}$ & {$[11.4,11.4,11.4]$} \\
$V_{b d}$ & {$[379.5,380.5,379]$} \\
$w_{0}$ & 314 \\
$I_{\text {line1d }}$ & -3.8 \\
$I_{\text {line2d }}$ & 7.6 \\
$V_{o q}$ & {$[0,0,0]$} \\
$I_{o q}$ & {$[0.4,-1.45,1.25]$} \\
$I_{l d}$ & {$[-5.5,-7.3,-4.6]$} \\
$V_{b q}$ & {$[-6,-6,-5]$} \\
$\delta$ & {$[0,1.9 e-3,-0.0113]$} \\
$I_{\text {line1q }}$ & 0.4 \\
$I_{\text {line2q }}$ & -1.3 \\
\hline
\end{tabular}

TABle 3: The multiple operating conditions.

\begin{tabular}{lcccc}
\hline Case & $R_{\text {load } 1}(\Omega)$ & $L_{\text {load } 1}(H)$ & $R_{\text {load } 3}(\Omega)$ & $L_{\text {load } 3}(H)$ \\
\hline Case 1 & 25 & $2.5 e-3$ & 20 & $2.2 e-3$ \\
Case 2 & 25 & $2.2 e-3$ & 20 & $2.5 e-3$ \\
Case 3 & 20 & $2.5 e-3$ & 25 & $2.2 e-3$ \\
Case 4 & 20 & $2.2 e-3$ & 25 & $2.5 e-3$ \\
\hline
\end{tabular}

TABLE 4: The limits of controller parameters.

\begin{tabular}{lcc}
\hline Variable & Lower limit & Upper limit \\
\hline$m_{p}$ & $9 e-6$ & $1 e-3$ \\
$n_{q}$ & $1.3 e-5$ & $9 e-3$ \\
$K_{p v}$ & 0.0003 & 0.1 \\
$K_{i v}$ & 200 & 450 \\
$K_{p c}$ & 0.01 & 15.5 \\
$K_{i c}$ & $10 e 3$ & $16 e 4$ \\
\hline
\end{tabular}


we can obtain the value domain of the variables from the root locus of the critical eigenvalues with the variable variation.

5.2. Optimization Method Verification. In the last subsection, the four operating conditions have been designed; and we implement the SQP-GS method in MATLAB to optimize the droop controller parameters in microgrid.

From Table 5, we can see the comparison of spectral abscissas between the unoptimized and the optimized values in the four operating conditions. In Case 1 and Case 3, the unoptimized spectral abscissa is 72.3025 and 72.3112 , respectively. It stands that the microgrid system is susceptible to encounter oscillation when random load fluctuation occurs. After the optimization, the optimized spectral abscissa is -16.1651 and -16.1212 , respectively. It proves that the small-signal stability of the microgrid in four operating conditions is enhanced through SQP-GS method optimization. Therefore, it demonstrated that the SQP-GS method is effective for optimizing the droop controller parameters.

The original parameters and the optimized parameters of the controller are given in Tables 6 and 7. As for the original parameters, the droop controller parameter is designed by the traditional method [15]. Also, the voltage and current controller parameters are selected based on the small-signal stability analysis $[9,10]$. For the closed-form spectral abscissa sensitivities of $K_{p v}, K_{i v}, K_{p c}$, and $K_{i c}$ are fairly small, these controller parameters stay the same nearly in the iteration process.

From Figure 2, there are two eigenvalues with the positive real part in the original system of Case 1. One positive eigenvalue is 72.3025 , and the other one is 15.9420 . It means that the original system is unstable. After optimizing the controller parameters by the SQP-GS method, all the optimized eigenvalues lie on the left plane. The spectral abscissa of Case 1 is -16.1651 . It proves that the stability of the system is improved after the optimization.

In Figure 3, the spectral abscissa of Case 1 decreases in the iteration process with the SQP-GS method. After 83 iterations, the spectral abscissa of Case 1 varies from 72.3025 to -16.1651 . It means that the microgrid system with optimized droop controller parameters is stable in the smallsignal stability sense.

In Figure 4, the upper limit of the spectral abscissa is varied from -15 to -16.061 in the iteration process. It means that the spectral abscissas of all the cases are less than -16.061 . The stability margin of the system is improved.

Figure 5 depicts the sampling radius variation, which is one of the convergence indicators in SQP-GS method. After 83 iterations, the sampling radius decreases from $10 e-1$ to $10 e-7$; and it proves that the SQP-GS method arrives at convergence.

5.3. Comparison with GA Method. The SQP-GS and GA methods are called nine times, respectively, for optimizing the droop controller parameters; and the mean value of $\bar{\eta}$, spectral abscissa in Case 4 , and the computing time are listed in Table 8. As the table shows, the mean value of $\bar{\eta}$ optimized
TABle 5: The spectral abscissa of microgrid.

\begin{tabular}{lcc}
\hline & \multicolumn{2}{c}{ Spectral abscissa } \\
& Unoptimized & SQP-GS \\
\hline Case 1 & 72.3025 & -16.1651 \\
Case 2 & 72.3024 & -16.1647 \\
Case 3 & 72.3112 & -16.1212 \\
Case 4 & 72.3110 & -16.1217 \\
\hline
\end{tabular}

TABLE 6: The original parameter of controller.

\begin{tabular}{lcccccc}
\hline DG & $m_{p}$ & $n_{q}$ & $K_{p v}$ & $K_{i v}$ & $K_{p c}$ & $K_{i c}$ \\
\hline DG1 & $6.45 e-4$ & $2.13 e-3$ & 0.0394 & 365 & 6.19 & 13241 \\
DG2 & $6.45 e-4$ & $2.13 e-3$ & 0.0394 & 365 & 6.19 & 13241 \\
DG3 & $6.45 e-4$ & $2.13 e-3$ & 0.0394 & 365 & 6.19 & 13241 \\
\hline
\end{tabular}

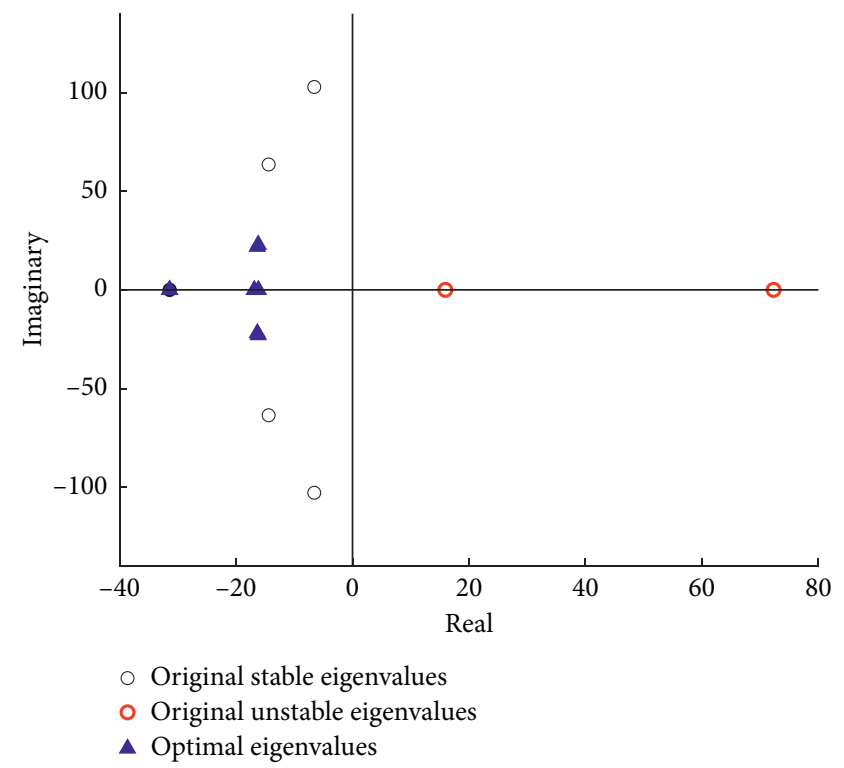

Figure 2: The comparison of eigenvalues of low frequency in Case 1.

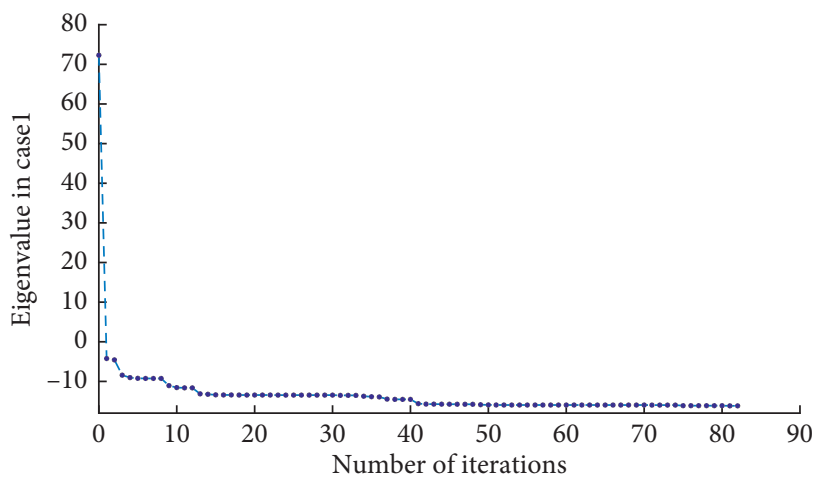

FIgURE 3: Spectral abscissa variation in the iteration process.

by the GA method is -15.31 . Besides, $\bar{\eta}$ optimized by the SQP-GS method is -16.06 . In the term of the spectral abscissa, the best value optimized by the GA method is -16.05 , while the value optimized by the SQP-GS method is -16.12. As for the computing time, the longest time for the 


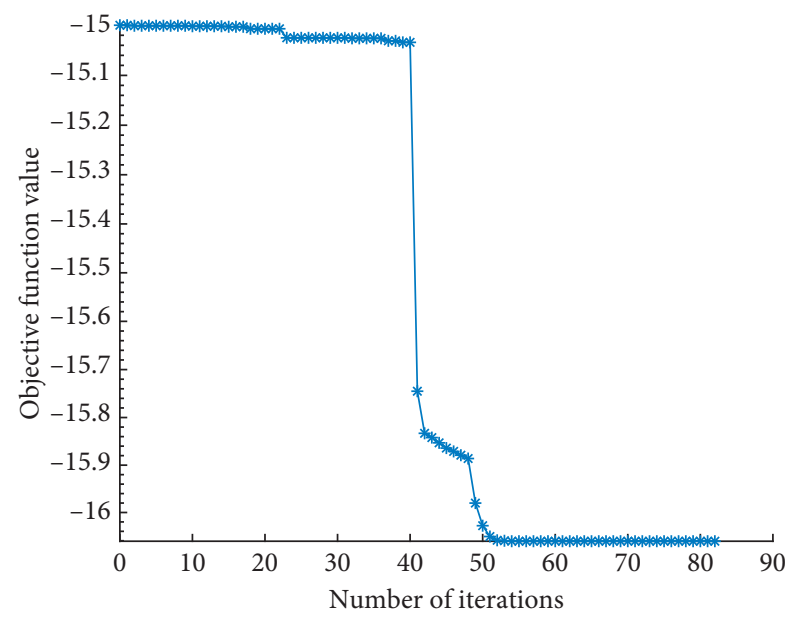

FIgURE 4: Objective function value variation in the iteration process.

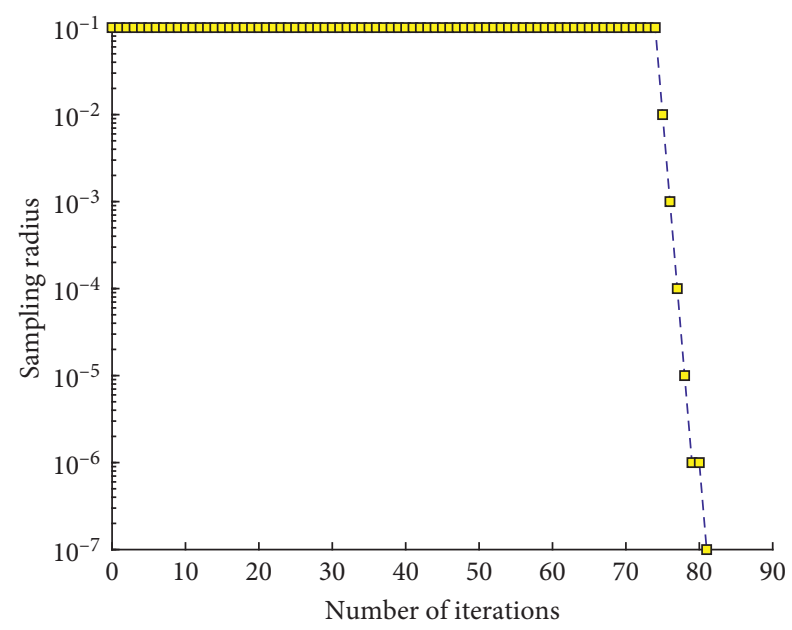

FIGURE 5: Sampling radius variation in the iteration process.

TABLE 7: The optimal parameters of controller.

\begin{tabular}{lcccccc}
\hline DG & $m_{p}$ & $n_{q}$ & $K_{p v}$ & $K_{i v}$ & $K_{p c}$ & $K_{i c}$ \\
\hline DG1 & $7.41 e-5$ & $1.95 e-4$ & 0.0397 & 365 & 6.19 & 13241 \\
DG2 & $1.45 e-5$ & $1.41 e-4$ & 0.0396 & 365 & 6.19 & 13241 \\
DG3 & $1.57 e-4$ & $7.97 e-4$ & 0.0398 & 365 & 6.19 & 13241 \\
\hline
\end{tabular}

TABLE 8: Comparison of spectral abscissa and computing time between GA method and SQP-GS method.

\begin{tabular}{lccccccc}
\hline & \multicolumn{4}{c}{ Spectral abscissa } & \multicolumn{3}{c}{ Computing time (h) } \\
Method & $\bar{\eta}$ & Best & Mean & Worst & Min & Mean & Max \\
\hline GA & -15.28 & -16.05 & -15.33 & -15.09 & 0.116 & 0.329 & 0.709 \\
SQP-GS & -16.06 & -16.12 & -16.12 & -16.12 & 0.095 & 0.097 & 0.102 \\
\hline
\end{tabular}

GA method to converge is 0.709 hours, while the shortest time is 0.116 hours. However, the max computing time of the SQP-GS method is 0.102 hours. These results demonstrate that the optimality and efficiency of the SQP-GS method are better than those of the GA method. Also, Table 8 shows that the SQP-GS can be globally convergent to stationary points with probability of one.

\section{Conclusion}

This paper proposes the SQP-GS method, which is a mathematical programming method to optimize the spectral abscissa of the microgrid. Combined with the proposed optimization model and SQP-GS method, the microgrid system is better robust in different operating conditions. Moreover, the SQP-GS method's effectiveness and efficiency are better than those of the GA method.

\section{Data Availability}

The microgrid system parameters and the initial condition of microgrid system which support the findings of this study are available in [15].

\section{Conflicts of Interest}

The authors declare that they have no conflicts of interest.

\section{Acknowledgments}

This work was supported in part by the National Natural Science Foundation of China under Grant no. 51967001, in part by the Natural Science Foundation of Guangxi under Grant no. 2018JJA160164, in part by Guangxi Special Fund for Innovation-Driven Development under Grant no. AA19254034, and in part by Guangxi Key Laboratory of Power System Optimization and Energy Technology Research Grant.

\section{References}

[1] D. E. Olivares, A. Mehrizi-Sani, A. H. Etemadi et al., "Trends in microgrid control," IEEE Transactions on Smart Grid, vol. 5, no. 4, pp. 1905-1919, 2014.

[2] C. Wei, Z. Shen, D. Xiao, L. Wang, X. Bai, and H. Chen, "An optimal scheduling strategy for peer-to-peer trading in interconnected microgrids based on ro and nash bargaining," Applied Energy, vol. 295, 2021 https://www.sciencedirect.com/ science/article/pii/S0306261921004888, Article ID 117024.

[3] Z. Li and M. Shahidehpour, "Small-signal modeling and stability analysis of hybrid ac/dc microgrids," IEEE Transactions on Smart Grid, vol. 10, no. 2, pp. 2080-2095, 2019.

[4] C. Wei, M. Benosman, and T. Kim, "Online parameter identification for state of power prediction of lithium-ion batteries in electric vehicles using extremum seeking," International Journal of Control, Automation and Systems, vol. 17, no. 11, pp. 2906-2916, 2019.

[5] T. V. Vu, S. Paran, F. Diaz-Franco, T. El-Mezyani, and C. S. Edrington, "An alternative distributed control architecture for improvement in the transient response of $\mathrm{dc}$ microgrids," IEEE Transactions on Industrial Electronics, vol. 64, no. 1, pp. 574-584, 2017.

[6] M. A. Hassan and M. A. Abido, "Optimal design of microgrids in autonomous and grid-connected modes using particle swarm optimization," IEEE Transactions on Power Electronics, vol. 26, no. 3, pp. 755-769, 2011. 
[7] B. K. Unnikrishnan, M. S. Johnson, and E. P. Cheriyan, "Small signal stability improvement of a microgrid by the optimised dynamic droop control method," IET Renewable Power Generation, vol. 14, no. 5, pp. 822-833, 2020.

[8] I. Chung, W. Liu, D. A. Cartes, E. G. Collins, and S. Moon, "Control methods of inverter-interfaced distributed generators in a microgrid system," IEEE Transactions on Industry Applications, vol. 46, no. 3, pp. 1078-1088, 2010.

[9] V. Naresh Kumar and S. K. Parida, "Parameter optimization of universal droop and internal model controller for multi inverter-fed dgs based on accurate small-signal model," IEEE Access, vol. 7, pp. 101 928-101 940, 2019.

[10] K. Yu, Q. Ai, S. Wang, J. Ni, and T. Lv, "Analysis and optimization of droop controller for microgrid system based on small-signal dynamic model," IEEE Transactions on Smart Grid, vol. 7, no. 2, pp. 695-705, 2016.

[11] C. Blum and A. Roli, "Metaheuristics in combinatorial optimization," ACM Computing Surveys, vol. 35, no. 3, pp. 268-308, 2003.

[12] M. O. Ball, "Heuristics based on mathematical programming," Surveys in Operations Research and Management Science, vol. 16, no. 1, pp. 21-38, 2011.

[13] F. E. Curtis and M. L. Overton, “A sequential quadratic programming algorithm for nonconvex, nonsmooth constrained optimization," SIAM Journal on Optimization, vol. 22, no. 2, pp. 474-500, 2012, https://doi.org/10.1137/ 090780201.

[14] P. Li, S. Huang, J. Qi, H. Wei, and X. Bai, "Optimal coordination of PSSs and pods by sequential quadratic programming with gradient sampling," in Proceedings of the 2020 IEEE/IAS Industrial and Commercial Power System Asia (I \& CPS Asia), pp. 604-610, Weihai, China, July 2020.

[15] N. Pogaku, M. Prodanovic, and T. C. Green, "Modeling, analysis and testing of autonomous operation of an inverterbased microgrid," IEEE Transactions on Power Electronics, vol. 22, no. 2, pp. 613-625, 2007.

[16] J. V. Burke and M. L. Overton, "Variational analysis of nonLipschitz spectral functions," Mathematical Programming, vol. 90, no. 2, pp. 317-351, 2001.

[17] A. S. Lewis and M. L. Overton, "Eigenvalue optimization," Acta Numerica, vol. 5, pp. 149-190, 1996.

[18] J. V. Burke, A. S. Lewis, and M. L. Overton, “A robust gradient sampling algorithm for nonsmooth, nonconvex optimization," Siam Journal on Optimization, vol. 15, no. 3, pp. 751-779, 2005.

[19] P. Li, Y. Wei, J. Qi, X. Bai, and H. Wei, "A closed-form formulation of eigenvalue sensitivity based on matrix calculus for small-signal stability analysis in power system," Journal of Modern Power Systems and Clean Energy, pp. 1-10, 2020.

[20] J. Nocedal, "Updating quasi-Newton matrices with limited storage," Mathematics of Computation, vol. 35, no. 151, pp. 773-782, 1980. 